\title{
Caracterização da composição centesimal, físico-química, compostos bioativos e capacidade antioxidante da pimenta jalapenho (Capsicum annuum var. annuum
} jalapenho)

\author{
Characterization of the centesimal composition, physicochemical, bioactive compounds and \\ antioxidant capacity of jalapeno pepper (Capsicum annuum var. annuum jalapenho) \\ Caracterización de la composición centesimal, fisicoquímica, compuestos bioactivos y capacidad \\ antioxidante del chile jalapeño (Capsicum annuum var. annuum jalapenho)
}

Recebido: 09/02/2021 | Revisado: 12/02/2021 | Aceito: 22/02/2021 | Publicado: 28/02/2021

\author{
Carla Adriana Ferrari Artilha-Mesquita \\ ORCID: https://orcid.org/0000-0001-8165-4863 \\ Universidade Estadual de Maringá, Brasil \\ E-mail: c.artilha@yahoo.com.br \\ Grasiele Scaramal Madrona \\ ORCID: https://orcid.org/0000-0002-8837-8424 \\ Universidade Estadual de Maringá, Brasil \\ E-mail: grasiele@yahoo.com
}

\begin{abstract}
Resumo
A pimenta Capsicum spp. é uma especiaria utilizada de diversas formas na alimentação, in natura ou industrializada, e em várias culturas gastronômicas. O rico perfil bioativo e de propriedade antioxidante atribuídos as pimentas destaca a espécie Capsicum аппиит var. аппиит "jalapenho" por apresentar características sensoriais e nutricionais que favorecem seu consumo e processamento industrial. Este trabalho teve como objetivo principal caracterizar a pimenta jalapenho (Capsicum annuиm L. var. апnиum) em sua composição centesimal, físico-química, de compostos bioativos e capacidade antioxidante. Os resultados demonstraram que o fruto de pimenta jalapenho apresentou valor nutricional semelhante as pimentas de sua espécie e também de outras espécies. As análises físico-química expressam resultados desejáveis para o processamento de produtos derivados da pimenta, com acidez moderada $\left(0,41 \pm 0,01 \mathrm{~g}^{1} 100 \mathrm{~g}^{-1}\right) \mathrm{e}$ tonalidade incidindo entre o laranja e vermelha $\left(h^{\circ}=55,17\right)$. A atividade antioxidante foi demonstrada através dos métodos de DPPH, FRAP e ABTS para os extratos aquosos do fruto de pimenta jalapenho, com 360,00 $\pm 5,00 \mu \mathrm{M}$ ET. $\mathrm{g}^{-1}, 121,47 \pm 2,54 \mu \mathrm{M}$ ESF. $\mathrm{g}^{-1}$ e 107,40 $\pm 1,16 \mu \mathrm{M} \mathrm{ET} . \mathrm{g}^{-1}$, respectivamente, obtendo maior atividade pelos métodos FRAP e ABTS. Os compostos bioativos apresentaram teores de 474,64 $\pm 12,26$ mg EAG.100g ${ }^{-1}$ de $^{2}$ compostos fenólicos, $112,88 \pm 0,36 \mathrm{mg}$ EQ.100 ${ }^{-1}$ de flavonoides totais, $3,79 \pm 0,26 \mathrm{mg} \cdot 100 \mathrm{~g}^{-1}$ de $\beta$-caroteno e 2,72 \pm $0,19 \mathrm{mg} \cdot 100 \mathrm{~g}^{-1}$ de licopeno, demonstrando que a pimenta jalapenho é uma fonte expressiva destes compostos bioativos sendo uma matéria-prima de interesse para indústria de alimentos.
\end{abstract}

Palavras-chave: Carotenoides; Valor nutricional; Atividade antioxidante.

\begin{abstract}
Capsicum spp. is a spice used in different ways in food, fresh or industrialized, and in various gastronomic cultures. The rich bioactive profile and antioxidant properties attributed to peppers highlights the species Capsicum annuиm var. аппиит "jalapenho" for presenting sensory and nutritional characteristics that favor its consumption and industrial processing. The main aim of this work was to characterize the jalapeño pepper (Capsicum annuит L. var. аппиит) in its centesimal composition, physicochemical, bioactive compounds and antioxidant capacity. The results showed that the fruit of the jalapeño pepper presented a nutritional value similar to peppers of its species and also of other species. The physical-chemical analyzes express desirable results for the processing of products derived from pepper, with moderate acidity $\left(0.41 \pm 0.01 \mathrm{~g}^{\left.-100 \mathrm{~g}^{-1}\right)}\right.$ and hue between orange and red $\left(\mathrm{h}^{\circ}=55.17\right)$. The antioxidant activity was demonstrated by the methods of DPPH, FRAP and ABTS for the aqueous extracts of the jalapeño pepper fruit, with $360.00 \pm 5.00 \mu \mathrm{M} \mathrm{TE} . \mathrm{g}^{-1}, 121.47 \pm 2.54 \mu \mathrm{M} \mathrm{FSE} . \mathrm{g}^{-1}$ and $107.40 \pm 1.16 \mu \mathrm{M} \mathrm{TE} . \mathrm{g}^{-1}$, respectively, obtaining higher activity by the FRAP and ABTS methods. The bioactive compounds showed levels of $474.64 \pm 12.26 \mathrm{mg}$ GAE. $100 \mathrm{~g}^{-1}$ of phenolic compounds, $112.88 \pm 0.36 \mathrm{mg}$ QE. $100 \mathrm{~g}^{-1}$ of total flavonoids, $3.79 \pm 0.26 \mathrm{mg} .100 \mathrm{~g}^{-1} \beta-$ carotene and $2.72 \pm 0.19 \mathrm{mg} .100 \mathrm{~g}^{-1}$ lycopene, demonstrating that the jalapeno pepper is an expressive source of these bioactive compounds and is a raw material of interest for the food industry.
\end{abstract}

Keywords: Carotenoids; Nutritional value; Antioxidant activity. 


\section{Resumen}

Capsicum spp. es una especia utilizada de diferentes formas en la alimentación, fresca o industrializada, y en diversas culturas gastronómicas. El rico perfil bioactivo y las propiedades antioxidantes atribuidas a los pimientos destaca la especie Capsicum annuum var. annuum "jalapenho" por presentar características sensoriales y nutricionales que favorecen su consumo y procesamiento industrial. Este trabajo tuvo como objetivo principal caracterizar el chile jalapeño (Capsicum annuиm L. var. аппиит) en su composición centesimal, fisicoquímica, de compuestos bioactivos y capacidad antioxidante. Los resultados mostraron que el fruto del chile jalapeño mostró un valor nutricional similar al de los pimientos de su especie y también de otras especies. Los análisis físico-químicos expresan resultados deseables para el procesamiento de productos derivados del pimiento, con acidez moderada $\left(0.41 \pm 0.01 \mathrm{~g}^{\left.-100 \mathrm{~g}^{-1}\right)} \mathrm{y}\right.$ tonalidad entre naranja y rojo $\left(h^{\circ}=55.17\right)$. La actividad antioxidante se demostró mediante los métodos de DPPH,

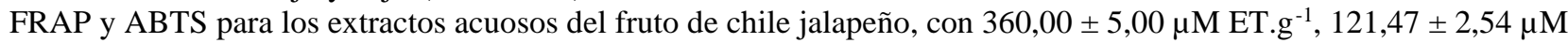
ESF. $\mathrm{g}^{-1}$ y $107,40 \pm 1,16 \mu \mathrm{M}$ ET. $\mathrm{g}^{-1}$, respectivamente, obteniendo mayor actividad por los métodos FRAP y ABTS. Los compuestos bioactivos mostraron niveles de $474.64 \pm 12.26 \mathrm{mg}$ EAG.100g ${ }^{-1}$ de compuestos fenólicos, $112.88 \pm$ $0.36 \mathrm{mg}$ EQ.100g ${ }^{-1}$ de flavonoides totales, $3.79 \pm 0.26 \mathrm{mg} .100 \mathrm{~g}^{-1} \beta$-caroteno y $2.72 \pm 0.19 \mathrm{mg} .100 \mathrm{~g}^{-1}$ licopeno, demostrando que el chile jalapeño es una fuente expresiva de estos compuestos bioactivos y es una materia prima de interés para la industria alimentaria.

Palabras clave: Carotenoides; Valor nutricional; Actividad antioxidante.

\section{Introdução}

Os frutos de pimentas (Capsicum spp.) são especiarias apreciadas amplamente no mundo e na cultura gastrônomica como ingredientes para fins alimentícios (Soldan, 2020), nutracêuticos (Bogusz et al., 2018), medicinais (Barduzzi, 2011; Soldan, 2020), defesa química (Razuck \& Razuck, 2020), propriedades farmacológicas e cosméticas (Carneiro et al., 2020).

Originárias das Américas, as pimentas se expandiram para outras regiões do mundo a partir do século XVI, entre as populações europeias e os povos indígenas (Rufino \& Penteado, 2006). As pimentas são pertencentes à família Solanaceae, atualmente, as espécies de pimentas do gênero Capsicum abrangem 35 espécies, com base na classificação taxonômica (Carrizo García et al., 2016), e de acordo com o nível de domesticação, apenas cinco são largamente cultivadas e utilizadas pelo homem: Capsicum annuum; C. bacccatum; C. chinense; $C$. frutescens e C. pubescens, sendo que a C. pubescens não é cultivada no Brasil.

O grupo varietal Capsicum anпиит var. annuит "jalapenho", popularmente conhecida como pimenta-jalapenho ou simplesmente jalapenho possui o fruto bem definido e robusto, com formato cônico, de cor avermelhada quando madura e com peculiares estrias brancas distribuídas nas paredes grossas de seu corpo, apresenta pungência média 30.000 a 40.000 SHU (Longatti, 2019; Gomes et al., 2019), com aroma ligeiramente frutado e é uma das variedades mais utilizadas para processamento industrial. Seu consumo alimentar acontece da forma convencional e por indústrias alimentícias que as processam como ingrediente, desde o uso como pimenta in natura, no formato de polpas (moídas, trituradas, com ou sem sementes) puras, fermentadas ou em salmouras; pimentas desidratadas; pimentas defumadas, em conservas, congeladas, em pó e como constituinte nas formulações, na forma de aromas (extratos hidroalcóolicos e oleoresinas), corantes, condimentos preparados, e principalmente, na forma de molhos.

As pimentas do gênero Capsicum são consideradas como fontes de compostos bioativos e de atividade antioxidante, como carotenoides, compostos fenólicos, vitamina C e capsaicinoides (Zhuang et al., 2012; Loizzo et al., 2015), e ao que se sabe, há pouco conhecimento disponível sobre a atividade antioxidante e dos compostos bioativos presentes em pimentas jalapenho (Capsicum annuum var. апnиum “jalapenho"). Os antioxidantes são capazes de neutralizar os radicais livres e prevenir as ações envolvidas nos processos de oxidação. Além disso, ácidos fenólicos, flavonoides e terpenos são os principais compostos bioativos que tem relação com as atividades antioxidantes e anti-inflamatórias encontradas em ervas e especiarias (Rubió, Motilva, \& Romero, 2013). Vale ressaltar que os níveis destes compostos podem variar de acordo com o genótipo, estágios de maturação e condições durante o crescimento e pós-colheita (Meckelmann et al., 2015; Bortolin et al., 2016; Bogusz et al., 2018). 
Alguns autores mencionam a pimenta jalapenho como fonte compostos fenólicos, vitaminas C, carotenoides, flavonoides e capsaicinoides (Loizzo et al., 2015; Longatti, 2019; Marti et al. 2011), de forma que seu prestígio está associado com a qualidade sensorial e nutricional dos frutos que são parâmetros relacionados ao conteúdo de compostos bioativos em termos de modificação de cor, aroma e sabor, além de proporcionar efeitos benéficos à saúde e prevenção de doenças relacionadas ao estresse oxidativo, cânceres e cardiopatites (Ornelas-Paz et al., 2010; Van Hung, 2016).

Neste contexto, diante das propriedades conhecidas na literatura em Capsicum spp., o objetivo deste trabalho foi avaliar as características da pimenta jalapenho (Capsicum annuum L. var. annuum) em sua composição centesimal, físicoquímica e do perfil bioativo, bem como determinar sua atividade antioxidante.

\section{Metodologia}

O estudo foi desenvolvido no Laboratório de Innovative Food Product, da Universidade Estadual de Maringá (UEM) entre o período de julho de 2019 a dezembro de 2020. A metodologia usada neste artigo se configura como uma pesquisa de natureza quantitativa e experimental por se tratar da coleta de dados numéricos através de medições de grandezas em suas respectivas unidades, utilizando-se metodologias específicas e analisadas estatisticamente para verificar a relação entre as variáveis (Pereira, Shitsuka, Parreira, \& Shitsuka, 2018).

\subsection{Matéria-prima}

A pimenta jalapenho (Capsicum annuum L. var. annuum) in natura foi adquirida de um produtor rural do Munícipio de Ainhumas, Estado de São Paulo, Brasil num total de $10 \mathrm{~kg}$. Os frutos da pimenta foram da safra de julho de 2019, em um lote único. Após a aquisição, os frutos foram selecionados em estágio de maturação fisiológica completa (maduras e vermelhas), classificados com a presença do pedúnculo para posteriormente serem extirpados (Figura 1), lavados com água potável, sanitizados com hipoclorito de sódio $\left(200 \mathrm{mg}\right.$. $\left.\mathrm{L}^{-1}\right)$ por imersão de 15 minutos, secas em papel absorvente, e posteriormente congeladas a $-18^{\circ} \mathrm{C}$ até o momento de sua utilização. Todos os reagentes utilizados foram de grau analítico.

Figura 1. Frutos de pimenta jalapenho (Capsicum annuum L. var. annuum) sem o pedúnculo.
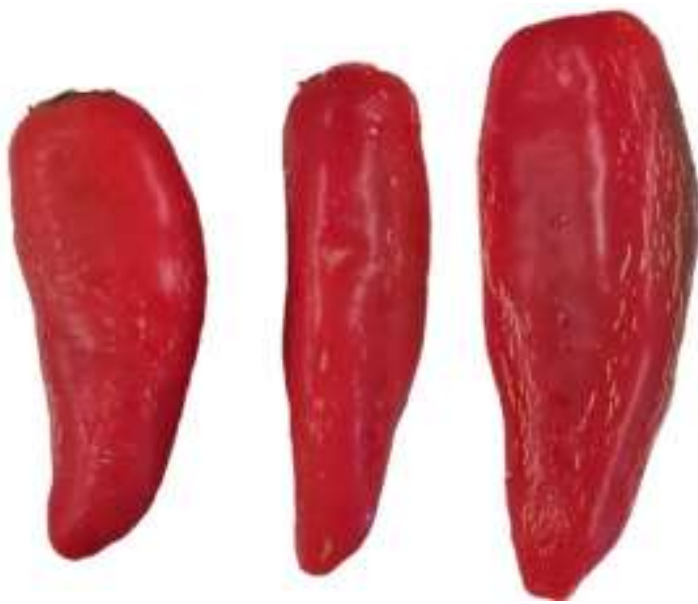

Fonte: Autores.

\subsection{Parâmetros de qualidade físico-químicas e composição centesimal}

As determinações analíticas de umidade, cinzas, proteínas (micro Kjeldahl), acidez, pH e sólidos solúveis foram realizadas de acordo com os métodos do IAL (2008). Os carboidratos foram quantificados por diferença, o teor de lipídios foi 
analisado conforme o método de extração de Bligh-Dyer (Cecchi, 1999). A determinação da atividade de água (aW) foi realizada em equipamento Aqualab®, Braseq ${ }^{\circledR}$, as determinações de cor foram realizadas em colorímetro portátil (Minolta CR400) pelo sistema CIEL $* \mathrm{a} * \mathrm{~b} *$. O ângulo $\mathrm{h}^{\circ}$ de tonalidade foi calculado com base na equação (1) (Arend et al., 2017). Todas as análises foram realizadas na pimenta triturada sem adição de água.

$$
\begin{aligned}
& \mathrm{h}^{\mathrm{o}}=\operatorname{tang}^{-1}\left(\mathrm{~b}^{*} / \mathrm{a}^{*}\right), \text { quando } \mathrm{a}^{*}>0 \mathrm{e}^{*} \geq 0 \text { ou } \\
& \mathrm{h}^{\mathrm{o}}=180+\operatorname{tang}^{-1}\left(\mathrm{~b}^{*} / \mathrm{a}^{*}\right), \text { quando } \mathrm{a}^{*}<0
\end{aligned}
$$

\subsection{Determinação da atividade antioxidante e compostos bioativos}

A determinação de compostos fenólicos totais foi realizada utilizando os reagentes Folin-Ciocalteu e carbonato de sódio $\left(\mathrm{Na}_{2} \mathrm{CO}_{3}\right)$ (Singleton \& Rossi, 1965; Pierpoint 2004). A absorbância foi verificada em espectrofotômetro (Femto, modelo 700 Plus) a $725 \mathrm{~nm}$ após 30 minutos de incubação a $25^{\circ} \mathrm{C}$. O ácido gálico foi utilizado como padrão para a curva de calibração. Os resultados foram expressos em $\mathrm{mg}$ de equivalente de ácido gálico (EAG).100 $\mathrm{g}^{-1}$ do produto. A atividade antioxidante pelo método de redução do radical estável DPPH (2,2-difenil-1-picrilhidrazila) foi determinada pelo método colorimétrico a $515 \mathrm{~nm}$ (Thaipong et al., 2006) em espectrofotômetro (Femto, modelo 700 Plus). O Trolox foi utilizado como padrão para a curva de calibração, os resultados foram expressos em $\mu \mathrm{M}$ de Trolox equivalente. $\mathrm{g}^{-1}$ produto. A eficiência da atividade sequestrante foi calculada de acordo com a equação 2, onde $\mathrm{A}_{\text {controle: }}$ Absorbância do controle negativo e $\mathrm{A}_{\text {amostra: }}$ : Absorbância média da amostra.

$$
\text { Eficiência do sequestro dos radicais livres }(\%)=\frac{\left(A_{\text {controle }}-A_{\text {amastra }}\right)}{A_{\text {controle }}} \times 100
$$

A capacidade antioxidante no método ABTS foi realizada utilizando um ensaio colorimétrico (Nenadis et al., 2004). A absorbância foi verificada em espectrofotômetro (Femto, modelo 700 Plus) a $734 \mathrm{~nm}$ após 6 minutos de incubação a $25^{\circ} \mathrm{C}$. Uma curva de calibração foi preparada utilizando uma solução padrão de trolox e os resultados expressos em $\mu \mathrm{M}$ Trolox equivalente. $\mathrm{g}^{-1}$ produto.

O poder antioxidante de redução de ferro (FRAP) foi realizado segundo Thaipong et al. (2006). A absorbância foi verificada em espectrofotômetro (Femto, modelo 700 Plus) a $595 \mathrm{~nm}$ e os resultados foram expressos em $\mu \mathrm{M}$ sulfato ferroso. $\mathrm{g}$ ${ }^{1}$ produto.

A determinação dos flavonoides totais foi realizada pelo ensaio colorimétrico (Alothman, Bhat, \& Karim, 2009). A absorbância foi verificada em espectrofotômetro (Femto, modelo 700 Plus) a $510 \mathrm{~nm}$. A curva de calibração foi preparada usando uma solução padrão de quercetina e os resultados foram expressos em mg de quercetina equivalente. $100 \mathrm{~g}^{-1}$ de produto.

A determinação dos carotenoides foi pela metodologia de Rodriguéz-Amaya (2001). A absorbância foi verificada em espectrofotômetro (Femto, modelo 700 Plus) a 470nm para licopeno e a $450 \mathrm{~nm}$ para o betacaroteno. A equação 3 é utilizada para o cálculo da quantidade do licopeno e beta-caroteno nas amostras em $\mu \mathrm{g} \cdot \mathrm{g}^{-1} \mathrm{e}$ convertidas para $\mathrm{mg} \cdot 100 \mathrm{~g}^{-1}$, no qual $\mathrm{A}=$ absorbância da solução no comprimento de onda de $470 \mathrm{~nm}$ para o licopeno e de $450 \mathrm{~nm}$ para o betacaroteno, $\mathrm{V}=$ volume final da solução; $A_{1 \epsilon m}^{196}=$ coeficiente de extinção ou coeficiente de absortividade molar de um pigmento em um determinado solvente específico (3450 para o licopeno e 2592 para o betacaroteno) e M= massa da amostra tomada para a análise.

$$
\frac{\mu g}{g}=\frac{A \cdot V \cdot 10^{6}}{A_{1 e m}^{196} \cdot M \cdot 100}
$$




\subsection{Análise estatística}

Todas as análises foram realizadas em triplicata e submetidas à análise estatística de variância e ao teste de Tukey para a diferença mínima significativa $(\mathrm{p}<0,05)$ entre as médias pelo o programa estatístico Sisvar 5.6. As curvas de calibração para as análises antioxidantes foram realizadas no programa GraphPrism 5.

\section{Resultados e Discussão}

\subsection{Caracterização da pimenta jalapenho}

A composição centesimal e físico-química dos frutos maduros de pimenta jalapenho (C. annuит var. annиum "jalapenho") em base úmida estão apresentadas na Tabela 1. A água constitui o principal componente do fruto (89,76\%) e os carboidratos a maior parte do extrato seco (7,47\%). Lutz e Freitas (2008) apresentaram valores de composição centesimal de pimenta jalapenho de $1,5 \mathrm{~g} \cdot 100 \mathrm{~g}^{-1}$ de proteínas, de $0,8 \mathrm{~g} \cdot 100 \mathrm{~g}^{-1}$ de lipídios, $10,4 \mathrm{~g} \cdot 100 \mathrm{~g}^{-1}$ de carboidratos e Mendoza-Sanchéz et al. (2015) encontraram para frutos maduros de pimenta jalapenho, o teor de umidade na faixa de $84,80 \mathrm{a} 89,90 \mathrm{~g}^{-100 \mathrm{~g}^{-1}}$, teor de cinzas de 0,50 g. $100 \mathrm{~g}^{-1}$ ligeiramente inferior ao do estudo e carboidratos entre 7,9 a 9,9 g.100g ${ }^{-1}$; Hwang et al. (2012) em análise de pimentões vermelhos (Capsicum апnиит L.) relataram níveis de 1,63g.100-1 de proteínas e 0,61g.100-1 de lipídios que são valores bem próximos para proteínas e lipídios e levemente maior de teor carboidratos. As análises dos constituintes apresentaram valores esperados para este o fruto de pimenta com valores similares aos dos estudos citados acima do mesmo fruto de espécie e variedade.

Tabela 1. Composição centesimal e parâmetros de qualidade físico-química da pimenta jalapenho.

\begin{tabular}{ll}
\hline Composição centesimal & Valor $\left(\mathrm{g} \cdot 100 \mathrm{~g}^{-1}\right)$ \\
\hline Umidade & $89,76 \pm 0,08$ \\
Cinzas & $0,68 \pm 0,02$ \\
Proteínas & $1,48 \pm 0,13$ \\
Carboidratos & $7,46 \pm 0,04$ \\
Lipídios & $0,62 \pm 0,04$ \\
\hline Composição físico-química $^{1}$ & Valor $\left(\mathrm{g} \cdot 100 \mathrm{~g}^{-1}\right)$ \\
\hline Acidez & $0,41 \pm 0,01$ \\
pH & $5,01 \pm 0,15$ \\
Atividade de água (aW) & $0,95 \pm 0,10$ \\
Sólidos solúveis & $8,26 \pm 0,07$ \\
\hline
\end{tabular}

Nota. Os dados são expressos como médias \pm desvios padrão. ${ }^{1}$ Expresso em gramas de ácido cítrico. $100 \mathrm{~g}^{-1}$.

Fonte: Autores.

A caracterização e o conhecimento dos atributos da composição físico-química são relevantes, pois contribuem com dados e informações a respeito do sabor e textura dos frutos de pimenta jalapenho. De acordo com os resultados, a pimenta jalapenho demonstra ser um fruto com acidez moderada e pH levemente ácido. Esta variável é de grande importância para a fabricação de molhos e conservas de pimenta, uma vez que eles são acidificados no final do processo para redução do $\mathrm{pH}$ e controle microbiológico, e, pimentas com pH mais ácido facilitam a manutenção da padronização desses produtos. Esses 
valores vêm de encontro com as faixas de valores encontrados em outros estudos desta pimenta com acidez de 0,36 a 0,48 g. $100 \mathrm{~g}^{-1}$, ph entre 4,84 a 5,36 (Ragassi et al., 2019).

O teor de sólidos solúveis apresentou valores um pouco acima dos resultados relatados pelos autores Ragassi et al. (2019) de 5,65 g.100 g e Gomes et al. (2019) de 7,50 g. $100 \mathrm{~g}^{-1}$, contudo, um fator importante a ser associado para o teor de sólidos solúveis e carboidratos é a data da colheita do fruto, que se relaciona com seu estágio de maturação, uma vez que uma colheita antecipada pode prejudicar o acúmulo de açúcares, visto que o teor de sólidos solúveis geralmente aumenta durante a maturação pela biossíntese ou degradação de polissacarídeos (Chitarra \& Chitarra, 2005).

A atividade de água representa a água livre presente nos alimentos e disponível para reações metabólicas. Para os frutos de pimenta jalapenho o valor foi de $0,95 \pm 0,01$ que é um valor significativamente elevado, visto que valores acima de 0,90 são considerados alimentos com alta atividade. Quando comparado com os valores citados por Mendoza-Sanchéz et al. (2015) de 0,97 a 0,98 em pimentas jalapenho maduras, observa-se uma pequena redução que segundo o autor pode estar relacionada as reações de ordem química e biológica, como a concentração de ácidos orgânicos envolvidos no ciclo de Krebs para o amadurecimento do fruto.

A leitura dos valores para os parâmetros de brilho $\left(\mathrm{L}^{*}\right)$ foi de $27,00 \pm 2,02$, a tendência para o vermelho (a *) foi de $22,81 \pm 0,58$ e para o amarelo $(\mathrm{b} *$ ) $32,79 \pm 2,18$. A tonalidade média dos frutos de pimentas foi definida pelo ângulo de cor " $\mathrm{h}$ " " situado no primeiro quadrante onde encontra-se a tonalidade vermelha com valor igual a 55,17\%. Ornela-Paz et al. (2010) obtiveram os parâmetros de ( $\left.\mathrm{a}^{*}\right)$ igual 41,30 e ( $\mathrm{b}^{*}$ ) de 40,90 para pimentas jalapenhos vermelhas in natura e a tonalidade com ângulo $h^{\circ}$ de 44,72. Borguini (2006) relatou faixas de ângulos de 54,78 a 71,98 para tomates orgânicos, que são frutos da família Solanaceae.

Figura 2. Corte longitudinal da pimenta jalapenho madura.

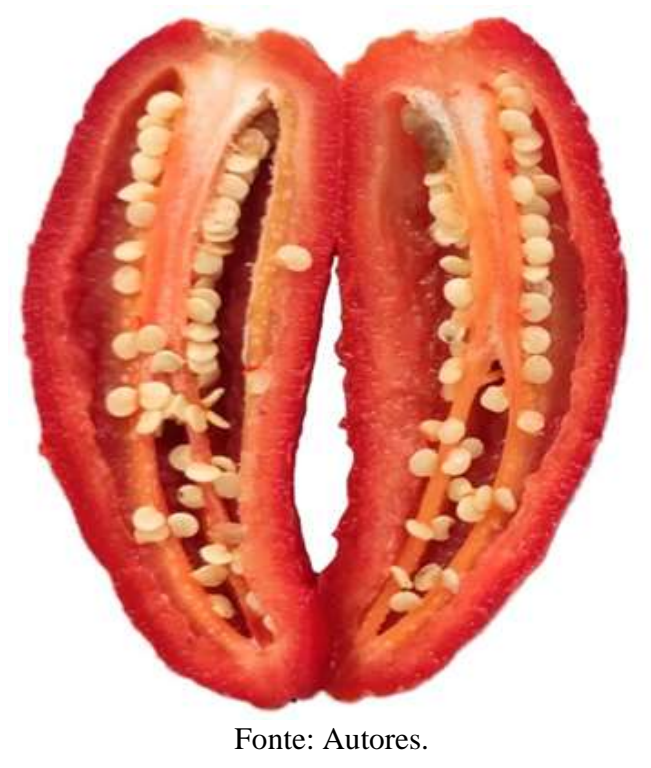

De acordo com o ângulo calculado, a tonalidade do fruto incidiu entre o laranja próximo ao vermelho. A cor é considerada um parâmetro importante para seleção de frutos e para a produção de molhos de pimenta jalapenho. Os frutos são consumidos verdes ou vermelhos, porém, quando os frutos estão maduros eles apresentam cor da polpa que varia do laranja ao vermelho (Figura 2) e cor externa vermelho-tijolo com brilho mais opaco que são atributos considerados ideais para o seu processamento. 


\subsection{Atividade antioxidante e compostos bioativos}

A concentração de compostos bioativos e a atividade antioxidante dos frutos maduros de pimenta jalapenho ( $C$. annuиm var. annuиm "jalapenho") em base úmida estão apresentados na Tabela 2. Os teores de carotenoides totais apresentaram 3,79 $\pm 0,26 \mathrm{mg} \cdot 100 \mathrm{~g}^{-1}$ de $\beta$-caroteno e 2,72 $\pm 0,19 \mathrm{mg} \cdot 100 \mathrm{~g}^{-1}$ de licopeno. Esses valores ficam acima aos valores informados por Gomes et al. (2014) que encontraram valores de $0,5 \mathrm{mg} \cdot 100 \mathrm{~g}^{-1} \mathrm{em}$ pimentas jalapenhos vermelhas cultivadas em campo, ao pleno sol; por Hwang et al. (2012) que obteve valores de $1,39 \mathrm{mg} / 100 \mathrm{~g}$ de $\beta$-caroteno em pimentões vermelhos (Capsicum annuиm L.) e Borguini (2006) que relatou 1,53 a $2,09 \mathrm{mg} \cdot 100 \mathrm{~g}^{-1} \mathrm{de}$ licopeno em tomates convencionais e orgânicos. Soldan (2020) também avaliou o teor de $\beta$-caroteno e licopeno em amostras de oleorresina de resíduos de pimenta jalapenho utilizando acetona como solvente, as amostras obtiveram teores de $18,69 \mathrm{mg} .100 \mathrm{~g}^{-1}$ de $\beta$-caroteno e $8,81 \mathrm{mg} .100 \mathrm{~g}^{-1}$ de licopeno (ambos em base seca). O $\beta$-caroteno é o mais difundido de todos os carotenoides nos alimentos, seja como constituinte secundário ou principal e o licopeno o principal pigmento de muitas frutas de polpa vermelha. Além disso, outras funções foram atribuídas a uma propriedade antioxidante dos carotenoides sendo que foi observado que o licopeno acíclico é mais eficaz do que o $\beta$-caroteno (Rodriguez-Amaya, 2001).

Tabela 2. Compostos bioativos e atividade antioxidante da pimenta jalapenho.

\begin{tabular}{ll}
\hline Compostos bioativos $^{1}$ & Valor \\
\hline Flavonoides totais & $112,88 \pm 0,36$ \\
Fenólicos Totais & $474,64 \pm 12,26$ \\
Carotenoides ( $\beta$-caroteno) & $3,79 \pm 0,26$ \\
Carotenoides (Licopeno) & $2,72 \pm 0,19$ \\
\hline Atividade Antioxidante ${ }^{2}$ & Valor \\
FRAP & $121,47 \pm 2,54$ \\
DPPH & $360,00 \pm 5,00$ \\
ABTS & $107,40 \pm 1,16$ \\
\hline
\end{tabular}

Nota. Os dados são expressos como médias \pm desvios padrão. ${ }^{1}$ Flavonoides totais expressos em mg eq. quercetina. $100 \mathrm{~g}^{-1}$; Fenólicos Totais expressos em mg EAG.100g ${ }^{-1}$; Carotenoides expressos em mg.100g ${ }^{-1}$. ${ }^{2} \mathrm{DPPH}$ expressos em $\mu \mathrm{M}$ equivalente de Trolox. $\mathrm{g}^{-1}$; ABTS expressos em $\mu \mathrm{M}$ equivalente de Trolox. $\mathrm{g}^{-1}$ e FRAP expressos em $\mu \mathrm{M}$ equivalente de sulfato ferroso.g $\mathrm{g}^{-1}$

Fonte: Autores.

Além dos carotenoides apontados neste trabalho, os autores Agostini-Costa et al. (2017) analisaram o teor de carotenoides totais e ácido ascórbico em dois cultivares vermelhos e três amarelos de pimenta jalapenha e dois cultivares de pimenta "red bode" e "red biquinho" ( $C$. chinense Jacquin) cultivadas em estufa e no campo durante o verão e a primavera. Os dados obtidos confirmaram a influência do genótipo e do meio ambiente nos compostos estudados, bem como identificaram dez carotenoides principais: $\beta$-caroteno, $\beta$-criptoxantina, zeaxantina, mutatoxantina, antheraxantina; capsantina, cis-capsantina, capsantina-5,6-epóxido, capsantona e capsorubina, destacando em maior quantidade a capsantina com $14,60 \mathrm{mg} \cdot 100 \mathrm{~g}^{1}$ e $\beta$ caroteno com $0,90 \mathrm{mg} \cdot 100 \mathrm{~g}^{-1}$.

A análise realizada nos extratos aquosos de pimenta jalapenho in natura revelou teor de 474,64 $\pm 12,26 \mathrm{mg}$ EAG.100 $\mathrm{g}^{-1}$ de compostos fenólicos totais, que são níveis elevados de fenólicos quando comparados com outras espécies do gênero Capsicum, como por exemplo a pimenta biquinho (Capsicum chinense) com teor de 41,31 mg EAG.100g-1 (Santos, 2018) e pimenta dedo de moça (Caspicum baccatum) com teores entre 150 a 264 mg EAG.100g ${ }^{-1}$ (Soethe, 2013) e 61,99 mg EAG.100g-1 (Sampaio et al., 2020), enquanto que Mendoza-Sánchez et al. (2015) relataram em seu trabalho teores entre 349,80 
a 480,30 mg EAG.100g-1 ${ }^{-1}$ de compostos fenólicos em pimentas jalapenho maduras, que são similares ao encontrado neste estudo e Ornelas-Paz et al. (2010) encontraram moderados teores de 160,94 mg EAG.100g-1 para pimenta jalapenho verde e 178,24 mg EAG.100g $\mathrm{g}^{-1}$ para pimenta jalapenho vermelha.

O conteúdo de flavonoides totais foi de 112,88 $\pm 0,36 \mathrm{mg} \mathrm{EQ} \cdot 100 \mathrm{~g}^{-1}$, podendo a pimenta jalapenho ser considerada uma boa fonte deste composto. Medina-Juárez et al. (2012) relataram conteúdos inferiores de $28,77 \mathrm{mg}$ EQ.100g $\mathrm{g}^{-1}$ para esta variedade e teores entre 25,38 a $60,36 \mathrm{mg}$ eq. quercetina. $100 \mathrm{~g}^{-1}$ para outras pimentas da mesma espécie (Capsicum annuит L.). Ueda (2013) descreveu 84,95 a 101,47 mg EQ.100g ${ }^{-1}$ destes compostos para pimenta dedo-de-moça (Capsicum baccatum) que é uma faixa de valores próxima da pimenta jalapenho. Existe uma escala de classificação botânica proposta pelos autores Peterson e Dwyer (1998) que especifica os alimentos conforme a concentração de flavonoides, teores entre 0,0001 a 0,039 $\mathrm{mg} \cdot \mathrm{g}^{-1}$ são considerados baixos, teores de 0,040 a $0,099 \mathrm{mg} \cdot \mathrm{g}^{-1}$ como moderados e teores maiores que $0,100 \mathrm{mg} \cdot \mathrm{g}^{-1}$ como altos. Por esta escala, os resultados obtidos para o fruto de pimenta jalapenho podem ser inferidos na classificação como alimentos de alta concentração de flavonoides

Essas variações entre o teor de compostos fenólicos totais e flavonoides podem ser explicadas devido as diferenças varietais entre as espécies de pimentas, mas também, a região demográfica, que influi com o solo e o clima, diferenças entre o cultivo, estádio de amadurecimento e manipulação pós-colheita (Silva et al., 2016; Meckelmann el al., 2015).

A atividade antioxidante é classificada como a capacidade dos compostos em inibir ou neutralizar os radicais livres e existem muitos métodos para sua determinação que podem diferir nos mecanismos de reação, aplicação e complexidade (Bogusz et al., 2018). Nos frutos de pimenta jalapenho a atividade antioxidante da pimenta jalapenho conseguiu inibir em apenas 43,09\% da quantidade total de radical livre DPPH (1,1-difenil-2-picrilhidrazil) caracterizando uma eficiência moderada. Medina-Juárez et al. (2012) relatou uma baixíssima eficiência $(8,45 \%)$ para pimenta jalapenho, enquanto que Dambros (2014) obteve uma inibição semelhante de 49,24\% para frutos da pimenta dedo-de-moça (C. baccatum), contudo, a mesma pimenta foi avaliada por Sampaio et al. (2020), que observaram valores superiores de $88,07 \%$ quando comparado com o presente estudo e o citado na literatura.

A capacidade antioxidante de redução do ferro (FRAP) apresentou atividade antioxidante de 121,47 $\pm 2,54 \mu \mathrm{M}$ eq. sulfato ferroso.g $\mathrm{g}^{-1}$. Em seu estudo, Boguz et al. (2018) avaliou a capacidade antioxidante de diferentes genótipos de pimentas observando uma alta atividade antioxidante pelo método FRAP (expressos em mg eq. ácido gálico) de $190,00 \mathrm{mg} .100 \mathrm{~g}^{-1} \mathrm{em} C$. frutescens, $169,00 \mathrm{mg} .100 \mathrm{~g}^{-1} \mathrm{em}$ C. chinense e $162,00 \mathrm{mg} .100 \mathrm{~g}^{-1} \mathrm{em} \mathrm{C}$. baccatum Pepper, e que foi aumentando conforme ocorria o amadurecimento nos dois anos de colheita.

Pelo método ABTS, a capacidade antioxidante da pimenta jalapenho apresentou maior atividade com 107,40 $\pm 1,16$ $\mu \mathrm{M}$ eq. Trolox. $\mathrm{g}^{-1}$, e quando comparadas com os resultados citados por Medina-Juárez et al. (2012) esses valores foram inferiores a $20 \mu \mathrm{M}$ eq. trolox . $\mathrm{g}^{-1}$ para pimenta jalapenha, 34,44 $\mu \mathrm{M}$ eq. Trolox $\mathrm{g}^{-1}$ e 33,60 $\mu \mathrm{M}$ eq. Trolox. $\mathrm{g}^{-1}$ para as pimentas Bell e Caribe, ambas da mesma espécie (Capsicum annuиm L.). Carvalho et al. (2015) estudando a atividade antioxidante de oito genótipos diferentes de pimenta, encontraram atividade variando de 46,79 a 113,08 $\mu \mathrm{M}$ eq. Trolox.g $\mathrm{g}^{-1}$, com destaque para o genótipo Capsicum annuиm L. com capacidade antioxidante de $83,59 \mu \mathrm{M}$ eq. Trolox. $\mathrm{g}^{-1}$. De acordo com os autores, o método ABTS é mais sensitivo em determinar a atividade de compostos lipofílicos e hidrofílicos, e neste estudo, a análise correlacionou-se positivamente com o conteúdo de compostos fenólicos ( $r=0,930 ; p<0,05)$.

Neste trabalho não foi verificado correlação significativa entre as análises realizadas para pimenta jalapenho. Inúmeros fatores podem ser relacionados com a ação dos compostos antioxidantes, como por exemplo, diferenças tanto na potência como na concentração de substâncias redutoras (Sim \& Sil, 2008), contudo, a composição química e as estruturas dos componentes ativos são fatores importantes que regulam a eficácia dos antioxidantes naturais (Abidille et al., 2005). 


\section{Considerações Finais}

$\mathrm{O}$ fruto de pimenta jalapenho demonstra possuir características nutricionais e físico-químicas que favorecem sua utilização no processamento para as indústrias alimentícias, e também se apresenta como uma expressiva fonte de compostos bioativos, manifestando teores de compostos fenólicos, flavonoides e carotenoides superiores a outras pimentas comparadas da mesma espécie e de espécies diferentes. A capacidade antioxidante da pimenta jalapenho foi demonstrada pelos métodos ABTS, DPPH e FRAP, demonstrando maior atividade para os métodos ABTS e FRAP.

São sugeridos estudos posteriores para avaliar a relação da atividade antioxidante com outros componentes fitoquímicos presentes no fruto e que podem desempenhar atividade antioxidante, como o ácido ascórbico (vitamina C) e os capsaicinoides.

\section{Conflito de Interesses}

Os autores declaram que a pesquisa foi conduzida na ausência de quaisquer relações comerciais ou financeiras que possam ser interpretados como um potencial conflito de interesse.

\section{Agradecimentos}

Agradecemos a Universidade Estadual de Maringá pelo apoio e estrutura para realização do projeto e a Coordenação de Aperfeiçoamento de Pessoal de Nível Superior (CAPES) pelo auxílio financeiro.

\section{Referências}

Abidille, M. D. H., Singh, R. P., Jayaprakasha, G. K., \& Jena, B. S. (2005). Antioxidant activity of the extracts from Dillenia indica fruits. Food Chemistry, 90 (4), 891-896. https://doi.org/10.1016/j.foodchem.2004.09.002

Alothman, M., Bhat, R., \& Karim, A. A. (2009). Antioxidant Capacity and Phenolic Content of Selected Tropical Fruits from Malaysia, Extracted with Different Solvents. Food Chemistry, 115, 785-88. http://dx.doi.org/10.1016/j.foodchem.2008.12.005

Agostini-Costa, T. S., Gomes, I. S., Melo, L. A. M., Reifschneider, F. J. B., \& Ribeiro, C. S. C. (2017). Carotenoid and total vitamin C content of peppers from selected Brazilian cultivars. Journal of Food Composition and Analysis, 57, 73-79. http://dx.doi.org/doi:10.1016/j.jfca.2016.12.020

Arend, G. D., Adorno, W., Rezzadori, K., Di Luccio, M., Chaves, V., Reginatto, F., \& Petrus, J. (2017). Concentration of phenolic compounds from strawberry (Fragaria X ananassa Duch) juice by nanofiltration membrane. Journal of Food Engineering, 201, 36-41. 10.1016/j.jfoodeng.2017.01.014.

Barduzzi, J. F. (2011). Extração e Quantificação da Capsaicina em Pimenta Dedo-de-moça. (Trabalho de conclusão de curso). Instituto Municipal de Ensino Superior -FEMA, Assis, SP, Brasil. https://cepein.femanet.com.br/BDigital/arqTccs/0811290529.pdf.

Bogusz, S. J., Libardi, S. H., Dias, F. F., Coutinho, J. P. Bochi, V. C., Rodrigues, D., Melo, A. M., \& Godoy, H. T. (2018). Brazilian Capsicum peppers: capsaicinoids content and antioxidant activity. Journal of the Science of Food and Agriculture, 98, 217-224. 10.1002/jsfa.8459

Borguini, R. G. (2006). Avaliação do potencial antioxidante e de algumas características físico-químicas do tomate (Lycopersicon esculentum) orgânico em comparação ao convencional. (Tese). Universidade de São Paulo, São Paulo, SP, Brasil. https://www.teses.usp.br/teses/disponiveis/6/6133/tde-14082006153722/pt-br.php.

Bortolin, R. C., Caregnato, F. F., Divan Junior, A. M., Zanotto-Filho, A., Moresco, K.S., Rios, A. O, Salvi, A. O., Ortmann, C. F., Carvalho, P., Reginatto, F. H., Gelain, D. P., \& Moreira, J. C. (2016). Chronic ozone exposure alters the secondary metabolite profile, antioxidant potential, anti-inflammatory property, and quality of red pepper fruit from Capsicum baccatum. Ecotoxicology Environmental Safety, 129, 16-24. 10.1016/j.ecoenv.2016.03.004

Carneiro, G. F. C., Lima, W. F., Mendes, G. S., Gomes, M. J. G., Mesquita, I. H. B., Silva Filho, D. R., Ximendes, J. A. F., Firmo, T. R., Ibiapina, S. I. O., Castro, E. B., Pereira, A. L., \& Ribeiro, L. M. (2020). Prospeção tecnológica das propriedades farmacológicas da pimenta (Capsicum). In: Tescarollo, I. L. (Org.). Farmácia e Promoção da Saúde. (pp. 94-101). Ponta Grossa: Atena. 10.22533/at.ed.2452003028

Carrizo García, C., Barfuss, M. H. J., Sehr, E. M., Barboza, G. E., Samuel, R., Moscone, E. A., \& Ehrendorfer, F. (2016). Phylogenetic relationships, diversification and expansion of chili peppers (Capsicum, Solanaceae). Annals of Botany, 118 (1), 35-51. https://doi.org/10.1093/aob/mcw079

Carvalho, A. V., Mattietto, R. A., Rios, A. O., Maciel, R. A., Moresco, K. S., \& Oliveira, T. C. S. (2015). Bioactive compounds and antioxidante activity of Pepper (Capsicum sp.) genotypes. Journal of Food Science and Technology, 52, 7457-7464. 10.1007/s13197-015-1833-0

Cecchi, H. M. (1999). Fundamentos teóricos e práticos em análise de alimentos: Unicamp.

Chitarra, M. I. F., \& Chitarra, A. D. (2005). Pós-colheita de frutas e hortaliças: fisiologia e manuseio (2a ed.): FAEPE. 
Dambros, J. I. (2014). Estabilidade de compostos potencialmente bioativos e alterações de qualidade em frutos e produtos de pimenta (Capsicum spp). (Dissertação). Faculdade de Agronomia Eliseu Maciel, Universidade Federal de Pelotas, RS, Brasil. http://www.dcta.create.inf.br/manag er/uploads/documentos/dissertacoes/dambros,_juliele_ilone._dissertacao_._2014_.pdf.

Gomes, I. S., Ribeiro, C. S. C., Reifscheneider, F. J. B., \& Agostini-Costa, T. S. (2014). Perfil de carotenoides em Capsicum annuum L. cv. jalapeño cultivados no campo, em pleno sol, ou em casa de vegetação, cultivo protegido. Congresso Brasileiro de Recursos Genéticos, Santos, SP, Brasil, 3. https://ainfo.cnptia.embrapa.br/digital/bitstream/item/113442/1/ResumoCBRG-371.pdf.

Gomes, L. M., Ribeiro, C. S. C., Ragassi, C. F., Silva, L. S., \& Reifschneider, F. J. B. (2019). Advanced lines of Jalapeño pepper with potential for mechanical harvesting. Ciência Rural, 49 (2). https://doi.org/10.1590/0103-8478cr20180222.

Hwang, I. G., Shin, Y. J., Lee, S., Lee, J., \& Yoo, S. M. (2012). Effects of different cooking methods on the antioxidant properties of red pepper (Capsicum annuит L.). Preventive Nutrition and Food Science, 17 (4), 286-292. http://dx.doi.org/10.3746/pnf.2012.17.4.286

Instituto Adolfo Lutz. (2008). Métodos físico-químicos para análise de alimentos (4a ed.). Instituto Adolfo Lutz. https://wp.ufpel.edu.br/nutricaobromatologia/files/2013/07/NormasADOLFOLUTZ.pdf.

Loizzo, M. R., Pugliese, A., Bonesi, M., Menichini, F., \& Tundis, R. (2015). Evaluation of chemical profile and antioxidant activity of twenty cultivars from Capsicum annum, Capsicum baccatum, Capsicum chacoense and Capsicum chinense: A comparison between fresh and processed peppers. Food Science and Technology, 64, 623-631. http://dx.doi.org/10.1016/j.lwt.2015.06.042

Longati, B. F. (2019). Melhoramento genético de pimenta do tipo Jalapeño (Capsicum annuum L.). (Tese). Universidade de São Paulo, Escola Superior de Agricultura "Luiz de Queiroz", Piracicaba, SP, Brasil. https://www.teses.usp.br/teses/disponiveis/11/11136/tde-22012020104830/publico/Bruna_Fernanda_Longatti.pdf.

Lutz, D. L., \& Freitas, S. C. (2008) Valor nutricional. In: Ribeiro, C. S. C., Lopes, C A., Henz, G. P., \& Reifschneider, F. J. (Eds). Pimentas Capsicum. (pp. 31-38). Brasília: Embrapa Hortaliças. http://ainfo.cnptia.embrapa.br/digital/bitstream/item/212748/1/Pimentas-Capsicum.pdf

Marti, M. C., Camejo, D., Vallejo, F., Romojaro, F., Bacarizo, S., Palma, J. M., Sevilla, F., \& Jiménez, A. (2011). Influence of fuit ripening stage and harvest period on the antioxidant content of sweet pepper cultivars. Plant Foods for Human Nutrition, 66, 416-423. 10.1007/s11130-011-0249-x

Meckelmann, S. W., Riegel, D.W., Zonneveld, M., Rios, L., Pena, K., Mueller-Seitz, E., \& Petz, M. (2015). Capsaicinoids, flavonoids, tocopherols, antioxidant capacity and color attributes in 23 native Peruvian chili peppers (Capsicum spp.) grown in three different locations. European Food Research and Technology, 240 (2), 273-283. 10.1007/s00217-014-2325-6

Medina-Juárez, L. A., Molina-Quijada, D. M., Toro-Sánchez, C. L, González-Aguilar, G. A., \& Gámez-Meza, N. (2012). Antioxidant activity of peppers (Capsicum annuum L.) extracts and characterization of their phenolic constituents. Interciencia, 37 (8), 588-593. https://www.redalyc.org/articulo.oa?id=339/33925396006

Mendoza-Sánchez, L. G. Mendoza-Lopez, M. R., Garcia-Barradas, O., Azuara-Nieto, E., Pascual-Pineda, L. A., \& Jiménez-Fernández, M. (2015). Physicochemical and antioxidant properties of jalapeño pepper (Capsicum annuum var. annuum) during storage. Revista Chapingo: Serie Horticultura, 21 (3), 229-241. 10.5154/r.rchsh.2015.06.010

Nenadis, N., Wang, L., Tsimidou, M., \& Zhang, H. (2004). Estimation of Scavenging Activity of Phenolic Compounds Using the ABTS+ Assay. Journal of Agricultural and Food Chemistry, 54, 4669-4674. https://doi.org/10.1021/jf0400056

Ornelas-Paz, J. J., Martínez-Burrola, J. M., Ruiz-Cruz, S., Santana-Rodríguez, V., Ibarra-Junquera, V., Olivas, G. I., \& Pérez-Martínez, J. D. (2010). Effect of cooking on the capsaicinoids and phenolics contents of Mexican peppers. Food Chemistry, 119, 1619-1625. https://doi.org/10.1016/j.foodchem.2009.09.054

Pereira, A. S., Shitsuka, D., Parreira, F. J., \& Shitsuka, R. (2018). Metodologia da Pesquisa Científica. UFSM. https://repositorio.ufsm.br/bitstream/ handle/1/15824/Lic_Computacao_Metodologia-Pesquisa-Cientifica.pdf?sequence=1

Peterson, J., \& Dwyer, J. (1998). Taxonomic classification helps identify flavonoid-containing foods on a semiquantitative food frequency questionnaire. Journal of the American Dietetic Association, 98 (6), 677-685. https://doi.org/10.1016/S0002-8223(98)00153-9

Pierpoint, W. S. (2004). The extraction of enzymes from plant tissues rich in phenolic compounds. Methods in Molecular Biology, 244, 65-74. https://doi.org/10.1385/1-59259-655-X:65

Ragassi, C. F., Zucolotto, J., Gomes, L. M., Ribeiro, C. S. C., Madeira, N. R., \& Reifschneider, F. J. B. (2019). Productivity, quality of fruits and architecture of Jalapeño pepper at different planting densities. Horticultura Brasileira, 37, 331-337. https://doi.org/10.1590/s0102-053620190312

Razuck, F. B., \& Razuck, R. C. S. (2020). Pulverizadores de agentes químicos - a química no gás lacrimogêneo e spray de pimenta. Revista Militar de Ciência e Tecnologia, 37 (1), 3-10. http://ebrevistas.eb.mil.br/CT/article/view/4417/3737

Rodriguéz-Amaya, D. B. (2001). A guide to carotenoid analysis in food: OMNI Reserarch ILSI Human Nutrition Institute. http://beauty-review.nl/wpcontent/uploads/2014/11/A-guide-to-carotenoid-analysis-in-foods.pdf.

Rubió, L., Motilva, M. J., \& Romero, M. P. (2013). Recent advances in biologically active compounds in herbs and spices: A review of the most effective antioxidant and anti-inflammatory active principles, Critical Reviews in Food Science Nutrition, 53, 943-953. http://dx.doi.org/10.1080/10408398.2011.574802

Rufino, J. L., \& Penteado, D. C. S. (2006). Importância econômica, perspectivas e potencialidades do mercado para pimenta. Informe Agropecuário, 27 (235), 30-39. https://www.bdpa.cnptia.embrapa.br/consulta/busca?b=ad\&id=780070\&biblioteca=CPATSA\&busca=autoria:\%22RUFINO,\%20J.\%20L.\%20dos\%20 S.\%22\&qFacets=autoria:\%22RUFINO,\%20J.\%20L.\%20dos\%20S.\%22\&sort=\&paginacao=t\&paginaAtual=1 
Research, Society and Development, v. 10, n. 2, e56410212785, 2021

(CC BY 4.0) | ISSN 2525-3409 | DOI: http://dx.doi.org/10.33448/rsd-v10i2.12785

Sampaio, P. H. O., Pereira, M. S., Dias, C. S., Rodrigues, L. M., Jacques, A. C., \& Crexi, V. T. (2020). Caracterização físico-química e compostos bioativos de pimentas (Capsicum baccatum var. pendulum). Simpósio de Segurança Alimentar, Rio Grande do Sul, Brasil, 7. http://schenautomacao.com.br/ssa7/envio/files/trabalho3_304.pdf.

Santos, A. S. (2018). Características agronômicas, físico-químicas e sensoriais de linhagens de pimenta biquinho cultivadas em sistema orgânico. (Dissertação). Universidade Federal de São Carlos, Araras, SP, 2018. https://repositorio.ufscar.br/handle/ufscar/9706?show=full.

Soethe, C. (2013). Caracterização físico-química e de compostos funcionais de pimenta dedo-de-moça 'BRS Mari' em função do estádio de maturação e tempo de armazenamento. (Dissertação). Universidade do Estado de Santa Catarina, Lages, SC, 2013. https://www.udesc.br/arquivos/cav/id_cpmenu/1347/Dissesrta_oCristinaSoetheCD_15676040535477_1347.pdf.

Soldan, A. C. F. (2020). Extração supercrítica de oleorresina de resíduo de pimento Capsicum annuum (jalapenho). (Dissertação). Universidade Federal de Uberlândia, Belo Horizonte, MG, 2020. https://repositorio.ufu.br/bitstream/123456789/29715/1/ExtracaoSupercriticaOleorresina.pdf.

Silva, R. P. F. F., Rocha-Santos, T. A. P., \& Duarte, A. C. (2016). Supercritical fluidextraction ofbioactivecompounds. TrACTrends in Analytical Chemistry, 76, 40-51. http://dx.doi.org/10.1016/j.trac.2015.11.013

Singleton, V. L., \& Rossi, J. A. (1965). Colorimetry of Total Phenolics with Phosphomolybdic-Phosphotungstic Acid Reagents. American Journal of Enology and Viticulture, 16 (3), 144-158. http://garfield.library.upenn.edu/classics1985/A1985AUG6900001.pdf

Thaipong, K., Boonprakob, U., Crosby, K., Cisneros-Zevallos, L., \& Byrne, D. H. (2006). Comparison of ABTS, DPPH, FRAP, and ORAC assays for estimating antioxidant activity from guava fruit extracts. Journal of Food Composition and Analysis, 19, 669-675. 10.1016/j.jfca.2006.01.003

Ueda, M. T. (2013). Compostos bioativos em pimentas: diferença entre variedades e efeito do cozimento. (Trabalho de conclusão de curso). Universidade Estadual Paulista "Júlio de Mesquita Filho", Araraquara, SP, 2013. https://repositorio.unesp.br/handle/11449/121636.

Van Hung, P. (2016) Phenolic compounds of cereals and their antioxidante capacity. Critical Reviews in Food Science and Nutrition, 56 (1), 25-35. https://doi.org/10.1080/10408398.2012.708909

Zhuang, Y., Chen, L., Sun, L., \& Cao, J. (2012). Bioactive characteristics and antioxidant activities of nine peppers. Journal of Functional Foods, 4, 331-338. 10.1016/j.jff.2012.01.001 